ITP-UU-13/32

SPIN-13/24

\title{
Twisted Heisenberg chain and the six-vertex model with DWBC
}

\author{
W. Galleas \\ Institute for Theoretical Physics and Spinoza Institute, \\ Utrecht University, Leuvenlaan 4, 3584 CE Utrecht, \\ The Netherlands \\ w.galleas@uu.nl
}

\begin{abstract}
In this work we establish a relation between the six-vertex model with Domain Wall Boundary Conditions (DWBC) and the $X X Z$ spin chain with anti-periodic twisted boundaries. More precisely, we demonstrate a formal relation between the zeroes of the partition function of the six-vertex model with DWBC and the zeroes of the transfer matrix eigenvalues associated with the six-vertex model with a particular nondiagonal boundary twist.
\end{abstract}

PACS numbers: 05.50+q, 02.30.IK

Keywords: Heisenberg chain, domain wall boundaries, functional equations 


\section{Contents}

1 Introduction $\quad 2$

2 Heisenberg chain and the DWBC partition function 3

3 Functional equations 5

4 The eigenvalues $\Lambda$ and the partition function $Z \quad 8$

4.1 The zeroes $w_{j} \ldots \ldots \ldots \ldots \ldots \ldots \ldots$

4.2 Truncation at roots of unity . . . . . . . . . . . . . 13

5 Concluding remarks $\quad 17$

6 Acknowledgements $\quad 17$

$\begin{array}{ll}\text { A The function } V_{i_{2 m}, \ldots, i_{1}}^{(2 m)} & 17\end{array}$

\section{Introduction}

One of the remarkable roles played by integrable systems is the establishment of connections between seemingly unrelated topics. For instance, although the relation between one-dimensional quantum spin chains and two-dimensional classical vertex models is nowadays clear, this remarkable relation has its origins in Lieb's observation that the ice model transfer matrix and the $X X X$ spin chain hamiltonian share the same eigenvectors [1]. In fact, this relation was only made clear by Baxter [2] whom showed that the logarithmic derivative of a two-dimensional vertex model transfer matrix gives rise to an one-dimensional quantum spin chain hamiltonian. This correspondence between quantum spin chains and classical vertex models is well established for lattice systems but we also have further connections emerging in the continuum limit. For instance, it is believed that the massless regimes of vertex models in the continuum are described by the critical properties of Wess-Zumino-Witten field theories [3].

As far as vertex models with Domain Wall Boundary Conditions (DWBC) [4] are concerned, we can not immediately associate an one-dimensional spin chain along the lines of [5]. Nevertheless, the six-vertex model with domain wall boundaries still exhibits interesting relations with the theory of classical integrable systems [6], special functions $[7,8]$ and enumerative combinatorics $[9]$. Moreover, in the recent paper $[10]$ we have shown that the partition function of the six-vertex model with DWBC corresponds to the null eigenvalue wave-function of a certain many-body hamiltonian operator.

On the other hand, the $X X Z$ spin chain with anti-periodic boundary conditions can be embedded in the transfer matrix of a $\mathcal{U}_{q}[\widehat{\mathfrak{s l}}(2)]$ invariant six-vertex model with a particular non-diagonal boundary twist along the same lines of $[5,11 \mid$. This particular spin chain has also been studied in $[12-15]$ and it was the first system tackled through the algebraic-functional method used in $|16-18|$ for partition functions with domain wall boundaries. This method has been refined in a series of papers and here we intend to 
report on a novel connection between the twisted Heisenberg chain and the six-vertex model with DWBC.

This paper is organized as follows. In Section 2 we briefly describe the transfer matrix embedding the $X X Z$ chain with anti-periodic boundary conditions and introduce the notation we shall use throughout this paper. In Section 3 we explore the Yang-Baxter algebra along the lines of $[10]$ in order to derive a functional equation relating the transfer matrix eigenvalues and the partition function of the six-vertex model with DWBC. The consequences of this functional equation is discussed in Section 4 and, in particular, we show how our results can be simplified when the anisotropy parameter is a root of unity. Concluding remarks are then discussed in Section 5 and the Appendix $\mathrm{A}$ is devoted to the derivation of our main result.

\section{Heisenberg chain and the DWBC partition func- tion}

In this section we shall give a brief description of the anisotropic Heisenberg chain with anti-periodic boundary conditions. This model consists of a spin- $\frac{1}{2}$ system and here we shall mostly adopt the conventions of [13]. The system hamiltonian $\mathcal{H}$ acts on the tensor product space $\mathbb{V}_{\mathcal{Q}} \cong\left(\mathbb{C}^{2}\right)^{\otimes L}$ and it reads

$$
\mathcal{H}:=\sum_{i=1}^{L}\left(\sigma_{i}^{x} \sigma_{i+1}^{x}+\sigma_{i}^{y} \sigma_{i+1}^{y}+\cosh (\gamma) \sigma_{i}^{z} \sigma_{i+1}^{z}\right) \quad \in \quad \operatorname{End}\left(\mathbb{V}_{\mathcal{Q}}\right) .
$$

In (2.1) we have employed the notation $\sigma_{i}=\mathbb{1}^{\otimes(i-1)} \otimes \sigma \otimes \mathbb{1}^{\otimes(L-i)}$ where $\sigma \in\left\{\sigma^{x}, \sigma^{y}, \sigma^{z}\right\}$ denotes the standard Pauli matrices and $\mathbb{1}$ stands for the identity matrix in End $\left(\mathbb{C}^{2}\right)$. As far as boundary terms are concerned, here we consider the following anti-periodic conditions:

$$
\sigma_{L+1}^{x}:=\sigma_{1}^{x} \quad \sigma_{L+1}^{y}:=-\sigma_{1}^{y} \quad \sigma_{L+1}^{z}:=-\sigma_{1}^{z} .
$$

Transfer matrix. The hamiltonian (2.1) corresponds to the logarithmic derivative of a six-vertex model transfer matrix with a particular boundary twist. Let $\mathbb{V}_{\mathcal{A}} \cong \mathbb{V}_{j} \cong$ $\mathbb{C}^{2}$ and consider the element $G_{\mathcal{A}}:=\left(\begin{array}{ll}0 & 1 \\ 1 & 0\end{array}\right) \in \operatorname{End}\left(\mathbb{V}_{\mathcal{A}}\right)$. Then consider the operator $\mathcal{R}_{\mathcal{A} j}: \mathbb{C} \rightarrow \operatorname{End}\left(\mathbb{V}_{\mathcal{A}} \otimes \mathbb{V}_{j}\right)$ and define the transfer matrix $T: \mathbb{C} \rightarrow \operatorname{End}\left(\mathbb{V}_{\mathcal{Q}}\right)$ as

$$
T(\lambda):=\operatorname{Tr}_{\mathcal{A}}\left[G_{\mathcal{A}} \prod_{1 \leq j \leq L}^{\longrightarrow} \mathcal{R}_{\mathcal{A} j}\left(\lambda-\mu_{j}\right)\right]
$$

where $\lambda, \mu_{j} \in \mathbb{C}$. The trace in $(\overline{2.3})$ is taken over the space $\mathbb{V}_{\mathcal{A}}$ while the matrix $\mathcal{R} \in$ $\operatorname{End}\left(\mathbb{V}_{1} \otimes \mathbb{V}_{2}\right)$ reads

$$
\mathcal{R}(\lambda):=\left(\begin{array}{cccc}
a(\lambda) & 0 & 0 & 0 \\
0 & b(\lambda) & c(\lambda) & 0 \\
0 & c(\lambda) & b(\lambda) & 0 \\
0 & 0 & 0 & a(\lambda)
\end{array}\right)
$$


The non-null entries of (2.4) corresponds to the functions: $a(\lambda):=\sinh (\lambda+\gamma), b(\lambda):=$ $\sinh (\lambda)$ and $c(\lambda):=\sinh (\gamma)$ for parameters $\lambda, \gamma \in \mathbb{C}$. In this way the hamiltonian $(2.1)$ is obtained from the relation $\left.\mathcal{H} \sim \frac{d}{d \lambda} \ln T(\lambda)\right|_{\substack{\lambda=0 \\ \mu_{j}=0}}$ and it is also worth mentioning that the $\mathcal{R}$-matrix (2.4) satisfies the standard Yang-Baxter equation [19]. In addition to that the matrix $G_{\mathcal{A}}$ fulfills the property $\left[\mathcal{R}, G_{\mathcal{A}} \otimes G_{\mathcal{A}}\right]=0$ ensuring that the transfer matrix (2.3) forms a commutative family.

Monodromy matrix. Let $\mathcal{T}_{\mathcal{A}}: \mathbb{C} \rightarrow \operatorname{End}\left(\mathbb{V}_{\mathcal{A}} \otimes \mathbb{V}_{\mathcal{Q}}\right)$ be the following operator

$$
\mathcal{T}_{\mathcal{A}}(\lambda):=\prod_{1 \leq j \leq L} \mathcal{R}_{\mathcal{A} j}\left(\lambda-\mu_{j}\right)
$$

which shall be referred to as monodromy matrix. As the $\mathcal{R}$-matrix $(2.4)$ satisfies the Yang-Baxter equation, one can show that the monodromy matrix (2.5) fulfills the following quadratic identity

$$
\mathcal{R}_{12}\left(\lambda_{1}-\lambda_{2}\right) \mathcal{T}_{1}\left(\lambda_{1}\right) \mathcal{T}_{2}\left(\lambda_{2}\right)=\mathcal{T}_{2}\left(\lambda_{2}\right) \mathcal{T}_{1}\left(\lambda_{1}\right) \mathcal{R}_{12}\left(\lambda_{1}-\lambda_{2}\right)
$$

The relation (2.6) is usually referred to as Yang-Baxter algebra and since $\mathbb{V}_{\mathcal{A}} \cong \mathbb{C}^{2}$, the monodromy matrix $\mathcal{T}_{\mathcal{A}}$ can be recasted as

$$
\mathcal{T}_{\mathcal{A}}(\lambda)=:\left(\begin{array}{ll}
A(\lambda) & B(\lambda) \\
C(\lambda) & D(\lambda)
\end{array}\right)
$$

with operators $A, B, C, D \in \operatorname{End}\left(\mathbb{V}_{\mathcal{Q}}\right)$. In this way we find that the transfer matrix $(2.3)$ simply reads $T(\lambda)=B(\lambda)+C(\lambda)$.

Domain wall boundaries. The $\mathcal{R}$-matrix $(\overline{2.4})$ encodes the statistical weights of a sixvertex model as discussed in [19]. However, one still needs to define appropriate boundary conditions in order to having a non-trivial partition function for the model. The case of DWBC for the six-vertex model was introduced on a square lattice of dimensions $L \times L$ by Korepin in [4]. More precisely, in [4] the author derives a recurrence relation for the partition function of the model which was subsequently solved by Izergin [20]. The aforementioned partition function then reads

$$
Z\left(\lambda_{1}, \ldots, \lambda_{L}\right)=\left\langle\overline{0}\left|\prod_{1 \leq j \leq L}^{\longrightarrow} B\left(\lambda_{j}\right)\right| 0\right\rangle,
$$

with vectors $|0\rangle$ and $|\overline{0}\rangle$ defined as

$$
|0\rangle:=\left(\begin{array}{l}
1 \\
0
\end{array}\right)^{\otimes L} \quad \text { and } \quad|\overline{0}\rangle:=\left(\begin{array}{l}
0 \\
1
\end{array}\right)^{\otimes L} .
$$


Highest/lowest weight vectors. The vectors $|0\rangle$ and $|\overline{0}\rangle$ defined in $(2.9)$ are respectively the $\mathfrak{s l}(2)$ highest and lowest weight vectors. The action of the entries of the monodromy matrix $(2.7)$ on those vectors are given as follows:

$$
\begin{array}{rlrl}
A(\lambda)|0\rangle & =\prod_{j=1}^{L} a\left(\lambda-\mu_{j}\right)|0\rangle & D(\lambda)|0\rangle & =\prod_{j=1}^{L} b\left(\lambda-\mu_{j}\right)|0\rangle \\
A(\lambda)|\overline{0}\rangle & =\prod_{j=1}^{L} b\left(\lambda-\mu_{j}\right)|\overline{0}\rangle & D(\lambda)|\overline{0}\rangle & =\prod_{j=1}^{L} a\left(\lambda-\mu_{j}\right)|\overline{0}\rangle \\
B(\lambda)|\overline{0}\rangle & =0 & C(\lambda)|0\rangle & =0 .
\end{array}
$$

\section{Functional equations}

The spectrum of the anti-periodic Heisenberg chain hamiltonian (2.1) can be obtained directly from the spectrum of the transfer matrix $(2.3)$. This is due to the fact that the hamiltonian $\mathcal{H}$ is given by the logarithmic derivative of the transfer matrix $T$, in addition to the property $[T(\lambda), T(\mu)]=0$ ensured by the relation (2.6). Thus one can shift the attention to the spectral problem associated with the transfer matrix $T$. In its turn, this problem can be tackled through the method introduced in $[13$ and subsequently extended in $[16-18,21]$. For that it is convenient to introduce some extra definitions and conventions.

Definition 1. Let $B\left(\lambda_{i}\right) \in \mathbb{V}_{\mathcal{Q}}$ be an off-diagonal element of the Yang-Baxter algebra as defined in (2.6). We then introduce the following notation for the product of n generators $B$,

$$
\left[\lambda_{1}, \ldots, \lambda_{n}\right]:=\prod_{1 \leq i \leq n} B\left(\lambda_{i}\right) .
$$

Remark 1. The property $B(\lambda) B(\mu)=B(\mu) B(\lambda)$, encoded in the relation (2.6), ensures that $\left[\lambda_{1}, \ldots, \lambda_{n}\right]$ is symmetric under the permutation of variables $\lambda_{i} \leftrightarrow \lambda_{j}$. Thus, when it is convenient, we shall also employ the simplified notation $\left[X^{1, n}\right]:=\left[\lambda_{1}, \ldots, \lambda_{n}\right]$ where $X^{i, j}:=\left\{\lambda_{k} \mid i \leq k \leq j\right\}$.

Next we recall that $T(\lambda)=B(\lambda)+C(\lambda)$ and consider the action of $T\left(\lambda_{0}\right)$ over the element $\left[X^{1, n}\right]$. For that the most lengthy computation is the term $C\left(\lambda_{0}\right)\left[X^{1, n}\right]$ which can be evaluated with the help of the commutation relations contained in (2.6). This computation has been performed in $[4,16]$ and we shall restrict ourselves to presenting only the final results. In this way we are left with the following expression,

$$
\begin{aligned}
T\left(\lambda_{0}\right)\left[X^{1, n}\right]= & {\left[X^{0, n}\right]+\sum_{1 \leq i \leq n}\left[X_{i}^{1, n}\right]\left(\Gamma_{0, i}^{i} A\left(\lambda_{0}\right) D\left(\lambda_{i}\right)+\Gamma_{i, 0}^{i} A\left(\lambda_{i}\right) D\left(\lambda_{0}\right)\right) } \\
& +\sum_{1 \leq i<j \leq n}\left[X_{i, j}^{0, n}\right]\left(\Omega_{i, j} A\left(\lambda_{i}\right) D\left(\lambda_{j}\right)+\Omega_{j, i} A\left(\lambda_{j}\right) D\left(\lambda_{i}\right)\right),
\end{aligned}
$$


where $X_{i}^{1, n}:=X^{1, n} \backslash\left\{\lambda_{i}\right\}$ and $X_{i, j}^{0, n}:=X^{0, n} \backslash\left\{\lambda_{i}, \lambda_{j}\right\}$. The coefficients $\Gamma_{j, k}^{i}$ and $\Omega_{i, j}$ in (3.2) explicitly read

$$
\begin{aligned}
\Gamma_{j, k}^{i} & :=\frac{c\left(\lambda_{k}-\lambda_{j}\right)}{b\left(\lambda_{k}-\lambda_{j}\right)} \prod_{\lambda \in X_{i}^{1, n}} \frac{a\left(\lambda_{k}-\lambda\right)}{b\left(\lambda_{k}-\lambda\right)} \frac{a\left(\lambda-\lambda_{j}\right)}{b\left(\lambda-\lambda_{j}\right)} \\
\Omega_{i, j} & :=\frac{c\left(\lambda_{j}-\lambda_{0}\right)}{a\left(\lambda_{j}-\lambda_{0}\right)} \frac{c\left(\lambda_{0}-\lambda_{i}\right)}{a\left(\lambda_{0}-\lambda_{i}\right)} \frac{a\left(\lambda_{j}-\lambda_{i}\right)}{b\left(\lambda_{j}-\lambda_{i}\right)} \prod_{\lambda \in X_{i, j}^{0, n}} \frac{a\left(\lambda_{j}-\lambda\right)}{b\left(\lambda_{j}-\lambda\right)} \frac{a\left(\lambda-\lambda_{i}\right)}{b\left(\lambda-\lambda_{i}\right)} .
\end{aligned}
$$

The interpretation of the Yang-Baxter algebra as a source of functional equations [10] can now be immediately invoked. In this way one can recognize (3.2) as a Yang-Baxter relation of order $n+1$ and, in addition to extra properties, this relation will allow us to derive a functional equation describing the spectrum of the transfer matrix $T$. For that it is also convenient to introduce the following definition.

Definition 2. Let $n \in \mathbb{Z}_{>0}$ be a discrete index and $\mathcal{M}(\lambda):=\{A, B, C, D\}(\lambda)$. Also, let $\mathcal{W}_{n}:=\mathcal{M}\left(\lambda_{1}\right) \times \mathcal{M}\left(\lambda_{2}\right) \times \ldots \mathcal{M}\left(\lambda_{n}\right)$ with n-tuples $\left(\xi_{1}, \xi_{2}, \ldots, \xi_{n}\right)$ being understood as $\prod_{1 \leq i \leq n} \xi_{i}$. Then consider the function space $\mathbb{C}\left[\lambda_{1}^{ \pm 1}, \ldots, \lambda_{n}^{ \pm 1}\right]$ of regular complex-valued functions on $\left(\lambda_{1}, \ldots, \lambda_{n}\right) \in \mathbb{C}^{n}$ and define $\tilde{\mathcal{W}}_{n}:=\mathbb{C}\left[\lambda_{1}^{ \pm 1}, \ldots, \lambda_{n}^{ \pm 1}\right] \otimes \operatorname{span}_{\mathbb{C}}\left(\mathcal{W}_{n}\right)$. The map $\pi_{n}$ is then introduced as the following $n$-additive continuous map

$$
\pi_{n}: \tilde{\mathcal{W}}_{n} \rightarrow \mathbb{C}\left[\lambda_{1}^{ \pm 1}, \lambda_{2}^{ \pm 1}, \ldots, \lambda_{n}^{ \pm 1}\right]
$$

In other words, the map $\pi_{n}$ associates a multivariate complex function to any product of $n$ generators of the Yang-Baxter algebra.

The next step within this approach consists in finding a suitable realization of the map $\pi_{n}$ which is able to convert $(\overline{3.2})$ into appropriate functional equations. We shall proceed along the lines of [10] and adopt a particular scalar product as realization of $\pi_{n}$.

Realization of $\boldsymbol{\pi}_{\boldsymbol{n}}$. Let $|\Psi\rangle \in \operatorname{span}\left(\mathbb{V}_{\mathcal{Q}}\right)$ be an eigenvector of the transfer matrix $(2.3)$ with eigenvalue $\Lambda(\lambda)$. More precisely, we have the action $T(\lambda)|\Psi\rangle=\Lambda(\lambda)|\Psi\rangle$. Then, taking into account the definition $(2.9)$, we define the map $\pi$ as

$$
\pi_{n+1}(\mathcal{A}):=\langle\Psi|\mathcal{A}| 0\rangle \quad \forall \mathcal{A} \in \tilde{\mathcal{W}}_{n+1}
$$

At this stage we have gathered all the ingredients required to convert the Yang-Baxter algebra relation (3.2) into a functional equation characterizing the eigenvalues $\Lambda$. For that we only need to apply the map (3.4) to the relation (3.2), taking into account the realization (3.5). This procedure can be effectively carried out by noticing that the LHS of (3.2) obeys the following reduction property $\pi_{n+1} \rightarrow \pi_{n}$,

$$
\pi_{n+1}\left(T\left(\lambda_{0}\right)\left[X^{1, n}\right]\right)=\Lambda\left(\lambda_{0}\right) \pi_{n}\left(\left[X^{1, n}\right]\right) .
$$


On the other hand, by applying the map (3.4) to (3.2), the terms in the RHS are of the following form: $\pi_{n+1}\left(\left[X^{0, n}\right]\right), \pi_{n+1}\left(\left[X_{i}^{1, n}\right] A\left(z_{1}\right) D\left(z_{2}\right)\right)$ for $\left(z_{1}, z_{2}\right) \in \operatorname{Sym}\left(\left\{\lambda_{0}, \lambda_{i}\right\}\right)$ and $\pi_{n+1}\left(\left[X_{i, j}^{0, n}\right] A\left(z_{1}\right) D\left(z_{2}\right)\right)$ for $\left(z_{1}, z_{2}\right) \in \operatorname{Sym}\left(\left\{\lambda_{i}, \lambda_{j}\right\}\right)$. The term $\pi_{n+1}\left(\left[X^{0, n}\right]\right)$ can not be significantly simplified, but for $\mathrm{Y} \in\left\{X_{i}^{1, n}, X_{i, j}^{0, n}\right\}$ and using (2.10), we find that

$$
\pi_{n+1}\left([\mathrm{Y}] A\left(z_{1}\right) D\left(z_{2}\right)\right)=\prod_{k=1}^{L} a\left(z_{1}-\mu_{k}\right) b\left(z_{2}-\mu_{k}\right) \pi_{n-1}([\mathrm{Y}]) .
$$

Our results so far can be written in a more convenient form with the help of the notation $\pi_{n}([X]):=\mathcal{F}_{n}(X)$ for a given set $X=\left\{\lambda_{k}\right\}$ of cardinality $n$. In this way, this procedure yields the following set of functional equations,

$$
\begin{aligned}
\Lambda\left(\lambda_{0}\right) \mathcal{F}_{n}\left(X^{1, n}\right)= & \mathcal{F}_{n+1}\left(X^{0, n}\right)+\sum_{1 \leq i \leq n} M_{i}^{(n)} \mathcal{F}_{n-1}\left(X_{i}^{1, n}\right) \\
& +\sum_{1 \leq i<j \leq n} N_{j, i}^{(n)} \mathcal{F}_{n-1}\left(X_{i, j}^{0, n}\right)
\end{aligned}
$$

with coefficients $M_{i}^{(n)}=M_{i}^{(n)}\left(\vec{X}^{0, n}\right)$ and $N_{j, i}^{(n)}=N_{j, i}^{(n)}\left(\vec{X}^{0, n}\right)$ given by

$$
\begin{aligned}
M_{i}^{(n)} & :=\Gamma_{0, i}^{i} \prod_{k=1}^{L} a\left(\lambda_{0}-\mu_{k}\right) b\left(\lambda_{i}-\mu_{k}\right)+\Gamma_{i, 0}^{i} \prod_{k=1}^{L} a\left(\lambda_{i}-\mu_{k}\right) b\left(\lambda_{0}-\mu_{k}\right) \\
N_{j, i}^{(n)} & :=\Omega_{i, j} \prod_{k=1}^{L} a\left(\lambda_{i}-\mu_{k}\right) b\left(\lambda_{j}-\mu_{k}\right)+\Omega_{j, i} \prod_{k=1}^{L} a\left(\lambda_{j}-\mu_{k}\right) b\left(\lambda_{i}-\mu_{k}\right) .
\end{aligned}
$$

The symbol $\vec{X}^{0, n}$ has been introduced in order to emphasize that the functions $M_{i}^{(n)}$ and $N_{j, i}^{(n)}$ are not invariant under the permutation of all variables. Its precise definition is given as follows.

Definition 3. Let $i, j \in \mathbb{Z}$ such that $i<j$. Then $\vec{X}^{i, j}$ stands for the vector

$$
\vec{X}^{i, j}:=\left(\lambda_{i}, \lambda_{i+1}, \lambda_{i+2}, \ldots, \lambda_{j}\right) .
$$

For latter convenience we shall also define the symbols $\vec{X}_{k}^{i, j}$ and $\vec{X}_{k, l}^{i, j}$ for $i \leq k, l \leq j$ such that $k<l$. They are respectively defined as

$$
\begin{aligned}
\vec{X}_{k}^{i, j} & :=\left(\lambda_{i}, \lambda_{i+1}, \ldots, \lambda_{k-1}, \lambda_{k+1}, \ldots, \lambda_{j}\right) \\
\vec{X}_{k, l}^{i, j} & :=\left(\lambda_{i}, \lambda_{i+1}, \ldots, \lambda_{k-1}, \lambda_{k+1}, \ldots, \lambda_{l-1}, \lambda_{l+1}, \ldots, \lambda_{j}\right) .
\end{aligned}
$$

Some remarks are required at this stage. For instance, the functional equation (3.8) consists of an extension of the equation obtained in [13] and it has also been recently described in [15]. Moreover, at algebraic level there is no upper limit for the discrete index $n$ in Eq. (3.8). However, the $\mathfrak{s l}(2)$ highest weight representation theory imposes an upper bound for that index. 


\section{The eigenvalues $\Lambda$ and the partition function $Z$}

In the previous section we have derived a functional equation involving the eigenvalues $\Lambda$ of the transfer matrix $(2.3)$ and a certain set of functions $\mathcal{F}_{n}$. Here we intend to show that the functions $\mathcal{F}_{n}$ can be eliminated from the system of Eqs. (3.8) yielding a single equation for the eigenvalues $\Lambda$. The functional equation for $\Lambda$ obtained in this way will depend explicitly on the partition function $Z$ of the six-vertex model with DWBC.

Highest weight and domain walls. The highest weight representation theory of the $\mathfrak{s l}(2)$ algebra gives an upper bound for the number of operators $B$ entering the product (3.1) as discussed in $[4,16]$. This feature is manifested in the following property,

$$
\left[X^{1, L}\right]|0\rangle=Z\left(X^{1, L}\right)|\overline{0}\rangle
$$

Thus the relations $(4.1)$ and $(3.5)$ imply that $\mathcal{F}_{L}\left(X^{1, L}\right)=Z\left(X^{1, L}\right) \overline{\mathcal{F}}_{0}$ where $\overline{\mathcal{F}}_{0}=\langle\Psi \mid \overline{0}\rangle$. Moreover, the function $\mathcal{F}_{n}$ vanishes for $n>L$ due to $(2.10),(3.5)$ and (4.1). It is worth remarking here that Eq. (3.8) assumes that $\mathcal{F}_{n}$ vanishes for $n<0$.

In order to illustrate our procedure, let us firstly have a closer look at Eq. (3.8) for the case $L=2$. In that case we can set $n=0,1,2,3$ and by doing so we are left with the following set of equations:

$$
\begin{aligned}
\Lambda\left(\lambda_{0}\right) \mathcal{F}_{0} & =\mathcal{F}_{1}\left(X^{0,0}\right) \\
\Lambda\left(\lambda_{0}\right) \mathcal{F}_{1}\left(X^{1,1}\right) & =Z\left(X^{0,1}\right) \overline{\mathcal{F}}_{0}+M_{1}^{(1)} \mathcal{F}_{0} \\
\Lambda\left(\lambda_{0}\right) Z\left(X^{1,2}\right) \overline{\mathcal{F}}_{0} & =\sum_{1 \leq i \leq 2} M_{i}^{(2)} \mathcal{F}_{1}\left(X_{i}^{1,2}\right)+N_{2,1}^{(2)} \mathcal{F}_{1}\left(X_{1,2}^{0,2}\right) \\
0 & =\sum_{1 \leq i \leq 3} M_{i}^{(3)} Z\left(X_{i}^{1,3}\right)+\sum_{1 \leq i<j \leq 3} N_{j, i}^{(3)} Z\left(X_{i, j}^{0,3}\right) .
\end{aligned}
$$

The last equation in (4.2) involves solely the partition function $Z$ and it had been previously described in $[16]$. This single equation is fully able to determine the function $Z$, up to an overall constant factor, while the remaining equations relate the eigenvalue $\Lambda$ and the auxiliary function $\mathcal{F}_{1}$. We shall then use the first equation of $(4.2)$ to eliminate $\mathcal{F}_{1}$ from the second and third equations. By doing so we are left with the relations,

$$
\begin{aligned}
\Lambda\left(\lambda_{0}\right) \Lambda\left(\lambda_{1}\right) & =Z\left(X^{0,1}\right) k_{0}+M_{1}^{(1)} \\
\Lambda\left(\lambda_{0}\right)\left[Z\left(X^{1,2}\right) k_{0}-N_{2,1}^{(2)}\right] & =M_{1}^{(2)} \Lambda\left(\lambda_{2}\right)+M_{2}^{(2)} \Lambda\left(\lambda_{1}\right),
\end{aligned}
$$

where $k_{0}=\overline{\mathcal{F}}_{0} / \mathcal{F}_{0}$. In what follows we shall assume that the partition function $Z$ is already determined and the only unknown factors in $(4.3)$ are the coefficients $k_{0}$ and the function $\Lambda$. Both equations in $(\overline{4.3})$ are able to determine $k_{0}$ and the eigenvalues $\Lambda$, however, the first equation is non-linear while the second is linear. In fact, the direct inspection of (4.3) reveals that $k_{0}=\Lambda\left(\mu_{1}\right) \Lambda\left(\mu_{2}\right)\left[c^{2} a\left(\mu_{1}-\mu_{2}\right) a\left(\mu_{2}-\mu_{1}\right)\right]^{-1}$. 
For the case $L=3$ we can set $n=0,1,2,3,4$ in (3.8). Each choice produces an independent equation and the whole set consists of the following equations,

$$
\begin{aligned}
\Lambda\left(\lambda_{0}\right) \mathcal{F}_{0} & =\mathcal{F}_{1}\left(X^{0,0}\right) \\
\Lambda\left(\lambda_{0}\right) \mathcal{F}_{1}\left(X^{1,1}\right) & =\mathcal{F}_{2}\left(X^{0,1}\right)+M_{1}^{(1)} \mathcal{F}_{0} \\
\Lambda\left(\lambda_{0}\right) \mathcal{F}_{2}\left(X^{1,2}\right) & =Z\left(X^{0,2}\right) \overline{\mathcal{F}}_{0}+\sum_{1 \leq i \leq 2} M_{i}^{(2)} \mathcal{F}_{1}\left(X_{i}^{1,2}\right)+N_{2,1}^{(2)} \mathcal{F}_{1}\left(X_{1,2}^{0,2}\right) \\
\Lambda\left(\lambda_{0}\right) Z\left(X^{1,3}\right) \overline{\mathcal{F}}_{0} & =\sum_{1 \leq i \leq 3} M_{i}^{(3)} \mathcal{F}_{2}\left(X_{i}^{1,3}\right)+\sum_{1 \leq i<j \leq 3} N_{j, i}^{(3)} \mathcal{F}_{2}\left(X_{i, j}^{0,3}\right) \\
0 & =\sum_{1 \leq i \leq 4} M_{i}^{(4)} Z\left(X_{i}^{1,4}\right)+\sum_{1 \leq i<j \leq 4} N_{j, i}^{(4)} Z\left(X_{i, j}^{0,4}\right) .
\end{aligned}
$$

Similarly to the case $L=2$, we can eliminate the functions $\mathcal{F}_{i}$ from the system of equations (4.4) in favor of the function 1 . By carrying out this procedure recursively, we find that the third and fourth equations in (4.4) can be rewritten as

$$
\begin{aligned}
\Lambda\left(\lambda_{0}\right) \Lambda\left(\lambda_{1}\right) \Lambda\left(\lambda_{2}\right)= & Z\left(X^{0,2}\right) k_{0}+\Lambda\left(\lambda_{0}\right)\left[M_{1}^{(1)}\left(\vec{X}^{1,2}\right)+N_{2,1}^{(2)}\left(\vec{X}^{0,2}\right)\right] \\
& +\Lambda\left(\lambda_{1}\right) M_{2}^{(2)}\left(\vec{X}^{0,2}\right)+\Lambda\left(\lambda_{2}\right) M_{1}^{(2)}\left(\vec{X}^{0,2}\right) \\
\Lambda\left(\lambda_{0}\right) Z\left(X^{1,3}\right) k_{0}= & \sum_{1 \leq i \leq 3} M_{i}^{(3)}\left(\vec{X}^{0,3}\right) \prod_{\substack{k=1 \\
k \neq i}}^{3} \Lambda\left(\lambda_{k}\right)+\sum_{1 \leq i<j \leq 3} N_{j, i}^{(3)}\left(\vec{X}^{0,3}\right) \Lambda\left(\lambda_{0}\right) \prod_{\substack{k=1 \\
k \neq i, j \\
k}}^{3} \Lambda\left(\lambda_{k}\right) \\
& -\sum_{1 \leq i \leq 3} M_{i}^{(3)}\left(\vec{X}^{0,3}\right) M_{1}^{(1)}\left(\vec{X}_{i}^{1,3}\right)-\sum_{1 \leq i<j \leq 3} N_{j, i}^{(3)}\left(\vec{X}^{0,3}\right) M_{1}^{(1)}\left(\vec{X}_{i, j}^{0,3}\right) .
\end{aligned}
$$

In contrast to the case $L=2$, none of the equations in $(4.5)$ is linear and this is the general behavior for arbitrary $L$. At this stage it is also worth stressing that for both cases, namely $L=2$ and $L=3$, we have explicitly written two sets of equations relating the eigenvalues $\Lambda$ and the partition function $Z$. Each set is formed by two equations, i.e. (4.3) and (4.5), however, there is a dramatic difference between the first and the second equations of each set. For instance, while the first equation runs over the set of variables $\left\{\lambda_{k} \mid 0 \leq k \leq L-1\right\}$, the second equation is defined over the set $\left\{\lambda_{k} \mid 0 \leq k \leq L\right\}$. The partition function $Z$ can also be described through functional equations and a similar feature had previously appeared for that problem. If we compare the functional equations for $Z$ derived in $[16]$ and $[18]$ we can readily see they are defined over a different number of variables. In what follows we shall focus on the functional equation relating $\Lambda$ and $Z$ generalizing the first equation of $(\overline{4.3})$ and $(4.5)$ for arbitrary values of $L$. The following definitions will be helpful.

Definition 4. The symbol $[x]$ is defined as

$$
[x]:= \begin{cases}x & \text { for } x \in 2 \mathbb{Z}_{>0} \\ x-1 & \text { for } x \in\left(2 \mathbb{Z}_{>0}+1\right) .\end{cases}
$$


Definition 5. Let $f(\lambda) \in \mathbb{C}[\lambda]$ and consider the product operator $\widehat{\prod}_{\lambda}^{i_{1}, \ldots, i_{2 m}}: \mathbb{C}[\lambda] \rightarrow$ $\mathbb{C}\left[\lambda_{v_{1}}\right] \times \cdots \times \mathbb{C}\left[\lambda_{v_{L-2 m}}\right]$ for $\lambda_{v_{j}} \in X_{i_{1}, \ldots, i_{2 m}}^{0, L-1}$ such that $\lambda_{v_{j}} \neq \lambda_{v_{k}}$ if $j \neq k$. The relation $X_{i_{1}, \ldots, i_{n+1}}^{l, m}=X_{i_{1}, \ldots, i_{n}}^{l, m} \backslash\left\{\lambda_{i_{n+1}}\right\}$ generalizes recursively our previous definition and the product operator $\widehat{\prod}_{\lambda}^{i_{1}, \ldots, i_{2 m}}$ is defined as

$$
\left(\widehat{\prod}_{\lambda}^{i_{1}, \ldots, i_{2 m}} f\right)(\lambda):=\prod_{\lambda \in X_{i_{1}, \ldots, i_{2 m}}^{0, L-1}} f(\lambda)=\prod_{\substack{k=0 \\ k \neq i_{1}, \ldots, i_{2 m}}}^{L-1} f\left(\lambda_{k}\right) .
$$

Theorem 1. The partition function $Z$ can be written in terms of the eigenvalues $\Lambda$ according to the formula

$$
Z\left(X^{0, L-1}\right) k_{0}=\left\{\sum_{m=0}^{[L] / 2} \sum_{0 \leq i_{1}<\cdots<i_{2 m} \leq L-1} V_{i_{2 m}, \ldots, i_{1}}^{(2 m)} \widehat{\prod}_{\lambda}^{i_{1}, \ldots, i_{2 m}}\right\} \Lambda(\lambda)
$$

where

$$
\begin{aligned}
V_{i_{2 m}, \ldots, i_{1}}^{(2 m)}:=\sum_{J} \prod_{l=1}^{m} \prod_{n=1}^{L} a\left(\lambda_{j_{l}}-\mu_{n}\right) \prod_{\lambda \in X_{i_{1}, \ldots, i_{2 m}}^{0, L-1}} \frac{a\left(\lambda-\lambda_{j_{l}}\right)}{b\left(\lambda-\lambda_{j_{l}}\right)} \\
\quad \times \sum_{K(J)} \prod_{l=1}^{m} \prod_{n=1}^{L} b\left(\lambda_{k_{l}}-\mu_{n}\right) \frac{c\left(\lambda_{j_{l}}-\lambda_{k_{l}}\right)}{b\left(\lambda_{j_{l}}-\lambda_{k_{l}}\right)} \prod_{\lambda \in X_{i_{1}, \ldots, i_{2 m}}} \frac{a\left(\lambda_{k_{l}}-\lambda\right)}{b\left(\lambda_{k_{l}}-\lambda\right)} \\
\times \prod_{1 \leq r<s \leq m} \frac{a\left(\lambda_{k_{r}}-\lambda_{k_{s}}\right)}{b\left(\lambda_{k_{r}}-\lambda_{k_{s}}\right)} \frac{a\left(\lambda_{k_{r}}-\lambda_{j_{s}}\right)}{b\left(\lambda_{k_{r}}-\lambda_{j_{s}}\right)} \frac{a\left(\lambda_{k_{s}}-\lambda_{j_{r}}+\gamma\right)}{b\left(\lambda_{k_{s}}-\lambda_{j_{r}}\right)}
\end{aligned}
$$

with summation symbols defined as $\sum_{J}:=\sum_{\substack{j_{1}, \ldots, j_{m} \in \mathcal{I}_{2 m} \\ j_{1}<j_{2}<\cdots<j m}}$ and $\sum_{K(J)}:=\sum_{\substack{k_{1}, \ldots, k_{m} \in \mathcal{J}_{2 m} \\ k_{\alpha} \neq k_{\beta}}}$. The symbols $\mathcal{I}_{2 m}$ and $\mathcal{J}_{2 m}$ stand respectively for the sets $\mathcal{I}_{2 m}:=\left\{i_{1}, \ldots, i_{2 m}\right\}$ and $\mathcal{J}_{2 m}:=$ $\mathcal{I}_{2 m} \backslash\left\{j_{1}, \ldots, j_{m}\right\}$. For clarity's sake, we stress here that $V^{(0)}:=1$.

Proof. The proof follows from the extension of the derivation presented for the cases $L=2$ and $L=3$. These cases are respectively covered by formulae $(4.3)$ and $(4.5)$. The derivation of formula (4.9) for arbitrary $L$ is discussed in App. A.

Example 1. The RHS of (4.8) for $L=2$ reads

$$
\Lambda\left(\lambda_{0}\right) \Lambda\left(\lambda_{1}\right)+V_{1,0}^{(2)}
$$

while for $L=3$ we have

$$
\Lambda\left(\lambda_{0}\right) \Lambda\left(\lambda_{1}\right) \Lambda\left(\lambda_{2}\right)+\sum_{0 \leq i_{1}<i_{2} \leq 2} V_{i_{2}, i_{1}}^{(2)} \prod_{\substack{k=0 \\ k \neq i_{1}, i_{2}}}^{2} \Lambda\left(\lambda_{k}\right) .
$$


The structure of the relation (4.8) is quite appealing and some remarks are in order. For instance, the relation (4.8) converts the problem of evaluating the partition function $Z$ into the diagonalisation of the transfer matrix of the six-vertex model with a nondiagonal boundary twist. This situation is analogous to the case of the six-vertex model defined on a torus where the model partition function is given in terms of the eigenvalues of the standard transfer matrix of the six-vertex model with periodic boundary conditions $[19,22$. Furthermore, if one assumes that the eigenvalues $\Lambda$ are parameterized by solutions of Bethe ansatz like equations, as obtained in [12,14], then we could expect the relation $(4.8)$ to offer access to thermodynamic properties of the six-vertex model with DWBC in the same fashion as for the case with toroidal boundary conditions.

\subsection{The zeroes $\boldsymbol{w}_{j}$}

The eigenvalues $\Lambda(\lambda)$ are essentially a polynomial of order $L-1$ in the variable $x:=e^{2 \lambda}$ as demonstrated in [13]. Thus it can be written in terms of its zeroes $w_{j}$ as

$$
\Lambda(\lambda)=\Lambda(0) \prod_{j=1}^{L-1} \frac{\sinh \left(w_{j}-\lambda\right)}{\sinh \left(w_{j}\right)}
$$

We shall assume that the zeroes $w_{j}$ are all distinct and the following corollary will allow us to determine the zeroes $w_{j}$.

Corollary 1. The relation (4.8) under the specialization $\lambda_{j}=w_{j}$ for $1 \leq j \leq L-1$ implies the following constraints,

$$
\frac{Z\left(\lambda_{0}, w_{1}, \ldots, w_{L-1}\right)}{V_{L-1, \ldots, 0}^{([L])}\left(\lambda_{0}, w_{1}, \ldots, w_{L-1}\right)} k_{0}=\left\{\begin{array}{lll}
(-1)^{\frac{L}{2}} & \text { for } & L \in 2 \mathbb{Z}_{>0} \\
\Lambda\left(\lambda_{0}\right) & \text { for } & L \in\left(2 \mathbb{Z}_{>0}+1\right) .
\end{array}\right.
$$

Now we can use the analytic properties of the functions $Z$ and $V_{L-1, \ldots 0}^{([L])}$, in addition to the relation $(4.13)$, to determine the set of zeroes $\left\{w_{j}\right\}$. For that it will be important to notice that the variable $\lambda_{0}$ in $(4.13)$ is still an arbitrary complex variable. Moreover, the partition function $Z$ is a symmetric multivariate polynomial $[4,17]$, while the function $V_{L-1, \ldots, 0}^{([L])}$ for $L \in 2 \mathbb{Z}_{>0}$ consists of a polynomial of order $L-1$ in the variable $x_{0}:=e^{2 \lambda_{0}}$. On the other hand, for $L \in 2 \mathbb{Z}_{>0}+1$ we have

$$
V_{L-1, \ldots, 0}^{(L-1)}\left(\lambda_{0}, w_{1}, \ldots, w_{L-1}\right)=\frac{\tilde{V}_{L-1, \ldots, 0}^{(L-1)}\left(\lambda_{0}, w_{1}, \ldots, w_{L-1}\right)}{\prod_{j=1}^{L-1} b\left(\lambda_{0}-w_{j}\right)},
$$

where the function $\tilde{V}_{L-1, \ldots, 0}^{(L-1)}\left(\lambda_{0}, w_{1}, \ldots, w_{L-1}\right)$ is a polynomial of order $L-1$ in the variable $x_{0}$.

The case $\boldsymbol{L} \in \mathbf{2} \mathbb{Z}_{>\mathbf{0}}$. This case corresponds to even values of $L$ and we can use the analytical properties of $(4.13)$ to characterize the set of variables $\left\{w_{j}\right\}$. For this analysis it is important to recall that both functions $Z$ and $V_{L-1, \ldots, 0}^{(L)}$ in the LHS of $(4.13)$ are 
polynomials of the same degree in the variable $x_{0}$. Then, since the RHS of (4.13) is a constant, we can conclude that the residues of the LHS must vanish at the zeroes of $V_{L-1, \ldots, 0}^{(L)}$. In other words, the zeroes of $Z$ and $V_{L-1, \ldots, 0}^{(L)}$ must coincide. This analysis yields a formal condition determining the set $\left\{w_{j}\right\}$ which is summarized in Corollary 2 .

Corollary 2. Consider $L \in 2 \mathbb{Z}_{>0}$ and let $\lambda_{k}^{Z} \in\left\{\lambda \in \mathbb{C} \mid Z\left(\lambda, w_{1}, \ldots, w_{L-1}\right)=0\right\}$ while $\lambda_{k}^{V} \in\left\{\lambda \in \mathbb{C} \mid V_{L-1, \ldots, 0}^{(L)}\left(\lambda, w_{1}, \ldots, w_{L-1}\right)=0\right\}$. The zeroes $\lambda_{k}^{Z}$ and $\lambda_{k}^{V}$ shall depend on the set of parameters $\left\{w_{j}\right\}$ and we can conclude that

$$
\lambda_{k}^{Z}\left(\left\{w_{j}\right\}\right)=\lambda_{k}^{V}\left(\left\{w_{j}\right\}\right) \quad 1 \leq k \leq L-1 .
$$

The direct inspection of (4.15) for small values of $L$ reveals that the variables $w_{j}$ are completely fixed by the aforementioned constraints.

The case $L \in\left(2 \mathbb{Z}_{>0}+1\right)$. The situation for $L$ odd requires a slightly more elaborated analysis due to the presence of the eigenvalue $\Lambda$ in the RHS of (4.13). In that case we also need to consider (4.14), and it turns out that (4.13) simplifies to

$$
\frac{\Lambda(0)}{k_{0}} \prod_{j=1}^{L-1} b\left(-w_{j}\right)^{-1}=\frac{Z\left(\lambda_{0}, w_{1}, \ldots, w_{L-1}\right)}{\tilde{V}_{L-1, \ldots, 0}^{(L-1)}\left(\lambda_{0}, w_{1}, \ldots, w_{L-1}\right)} .
$$

The LHS of (4.16) is a constant with respect to the variable $\lambda_{0}$ while the RHS consists of a rational function. Thus the polynomials $Z$ and $\tilde{V}_{L-1, \ldots, 0}^{(L-1)}$ must share the same zeroes. Similarly to the $L$ even case, this statement can be formulated more precisely as the following corollary.

Corollary 3. Assume that $L \in\left(2 \mathbb{Z}_{>0}+1\right)$ and let $\lambda_{k}^{Z} \in\left\{\lambda \in \mathbb{C} \mid Z\left(\lambda, w_{1}, \ldots, w_{L-1}\right)=0\right\}$ as previously defined. Also, let $\tilde{\lambda}_{k}^{V} \in\left\{\lambda \in \mathbb{C} \mid \tilde{V}_{L-1, \ldots, 0}^{(L-1)}\left(\lambda, w_{1}, \ldots, w_{L-1}\right)=0\right\}$. Thus we have the following conditions determining the set of variables $\left\{w_{j}\right\}$,

$$
\lambda_{k}^{Z}\left(\left\{w_{j}\right\}\right)=\tilde{\lambda}_{k}^{V}\left(\left\{w_{j}\right\}\right) \quad 1 \leq k \leq L-1 .
$$

Both Corollaries 2 and 3 state that the zeroes of the partition function $Z$, with respect to one of its variables, coincide with the zeroes of the function $V_{L-1, \ldots, 0}^{([L])}$ when the remaining variables correspond to zeroes of the transfer matrix eigenvalues $\Lambda$.

Wronskian condition. The constraints $(4.15)$ and $(4.17)$ are given in terms of the zeroes of certain polynomials whose explicit evaluation might still be a very non-trivial problem. Alternatively, one can also obtain equations determining the set of zeroes $\left\{w_{j}\right\}$ in terms of the coefficients of the polynomial part of $V_{L-1, \ldots, 0}^{([L])}$ and $Z$. This analysis can be performed for $L \in 2 \mathbb{Z}_{>0}$ and $L \in\left(2 \mathbb{Z}_{>0}+1\right)$ in an unified manner with the help of the function $F$ defined as

$$
F:=\left\{\begin{array}{ll}
V_{L-1, \ldots, 0}^{(L)} & \text { for } L \in 2 \mathbb{Z}_{>0} \\
\tilde{V}_{L-1, \ldots, 0}^{(L-1)} & \text { for } L \in\left(2 \mathbb{Z}_{>0}+1\right)
\end{array} .\right.
$$


The term $\tilde{V}_{L-1, \ldots, 0}^{(L-1)}$ in $(4.18)$ has been previously defined in $(4.14)$. Thus the function $F\left(\lambda_{0}, w_{1}, \ldots, w_{L-1}\right)$ is a polynomial of order $L-1$ in the variable $x_{0}$ for $L \in \mathbb{Z}_{>0}$.

The equations fixing the zeroes $w_{j}$ from the coefficients of $Z$ and $F$ can be directly read from the relations (4.13) and (4.16). However, this approach would leave us with an overall constant factor and we can avoid this drawback by simply demanding that the Wronskian determinant between $Z$ and $F$ vanishes. This is justified by the fact that (4.13) and (4.16) tell us that $Z$ and $F$ and two linearly dependent functions. In this way we are left with the condition,

$$
P\left(x_{0}\right):=Z\left(x_{0},\left\{w_{j}\right\}\right) F^{\prime}\left(x_{0},\left\{w_{j}\right\}\right)-F\left(x_{0},\left\{w_{j}\right\}\right) Z^{\prime}\left(x_{0},\left\{w_{j}\right\}\right)=0,
$$

where the symbol $(')$ denotes differentiation with respect to the variable $x_{0}$.

The function $P$ is a polynomial of order $[L]$ in the variable $x_{0}$ which must vanish in the entire complex domain according to the Wronskian condition $(4.19)$. The coefficients of $P$ are given by

$$
\mathcal{C}_{k}=\left.\frac{1}{k !} \frac{\partial^{k} P}{\partial x_{0}^{k}}\right|_{x_{0}=0},
$$

and we demand these coefficients to vanish in order to satisfy $(4.19)$. Thus we end up with the following formal condition fixing the zeroes $w_{j}$,

$$
\mathcal{C}_{k}\left(\left\{w_{j}\right\}\right)=0 \quad 0 \leq k \leq[L] .
$$

It is important to remark here that $(4.21)$ provides one or two more equations than variables $w_{j}$ to be determined. For $L$ even we have one more equation, while for $L$ odd we have two additional equations. Each equation is a non-linear algebraic equation and consequently we have a large number of solutions. This feature is similar to what one finds when solving standard Bethe ansatz equations. However, the direct inspection of the solutions of (4.21) for small values of $L$ reveals that these extra equations play the role of a filter keeping only solutions which actually describe the spectrum of the transfer matrix (2.3).

\subsection{Truncation at roots of unity}

Vertex models based on solutions of the Yang-Baxter equation can exhibit special properties when its anisotropy parameter satisfies certain root of unity conditions. For instance, Tarasov demonstrated in $[23$ that the property

$$
\prod_{0 \leq k \leq l-1} B(\lambda-k \gamma)=0
$$

holds for the $\mathcal{U}_{q}[\widehat{\mathfrak{s l}}(2)]$ invariant six-vertex model when the anisotropy parameter $\gamma$ obeys the condition $e^{2 l \gamma}=1$. The case $l=1$ is not illuminating for our present discussion as we can see from definitions $(2.5)$ and $(2.7)$ that both operators $B(\lambda)$ and $C(\lambda)$ are proportional to the factor $\left(e^{2 \gamma}-1\right)$. Consequently the transfer matrix $(2.3)$ is also proportional to that same quantity, and this implies that its eigenvalues trivially vanishes when we set $l=1$. The free-fermion point $\gamma=\mathrm{i} \pi / 2$ is, in its turn, covered by the case $l=2$ and we shall start our analysis with this case despite its triviality. We shall then consider the cases $l=3$ and $l=4$ separately before discussing the general case. 
$\boldsymbol{l}=\mathbf{2}$. Our goal here is to analyze the system of equations (3.8) taking into account the representation theoretic properties of the functions $\mathcal{F}_{n}\left(X^{1, n}\right)=\pi_{n}\left(\left[\lambda_{1}, \ldots, \lambda_{n}\right]\right)$. The property $(4.22)$ can then be used in a very natural way and for $l=2$ we have $[\lambda, \lambda-\gamma]=0$. More precisely, we can exploit this property by looking at (4.3) under the specialization $\lambda_{0}=\lambda$ and $\lambda_{1}=\lambda-\gamma$. By doing so we are left with the following functional relation,

$$
\begin{aligned}
\Lambda(\lambda) \Lambda(\lambda-\gamma) & =M_{1}^{(1)}(\lambda, \lambda-\gamma) \\
& =\prod_{k=1}^{L} \sinh \left(\lambda-\mu_{k}\right)^{2}-\prod_{k=1}^{L} \sinh \left(\lambda-\mu_{k}+\gamma\right) \sinh \left(\lambda-\mu_{k}-\gamma\right)
\end{aligned}
$$

It is worth remarking here that (4.23) corresponds to an analogous of the inversion relation proposed by Stroganov in [24]. Next we consider the representation (4.12) and set $\lambda=w_{i}$ in $(4.23)$. This procedure then yields the following equation determining the set of zeroes $\left\{w_{j}\right\}$,

$$
\prod_{k=1}^{L} \frac{\sinh \left(w_{i}-\mu_{k}+\gamma\right)}{\sinh \left(w_{i}-\mu_{k}\right)} \frac{\sinh \left(w_{i}-\mu_{k}-\gamma\right)}{\sinh \left(w_{i}-\mu_{k}\right)}=1 .
$$

As previously mentioned, the free-fermion point $\gamma=\mathrm{i} \pi / 2$ fits in the case $l=2$ and at this particular point we find that (4.24) simplifies to

$$
\prod_{k=1}^{L} \operatorname{coth}\left(w_{i}-\mu_{k}\right)^{2}=1 \text {. }
$$

We can now readily see that $(4.25)$ generalizes the proposal of 15 in the presence of inhomogeneities $\mu_{k}$.

$\boldsymbol{l}=3 . \quad$ In that case the property $(4.22) \operatorname{reads}[\lambda, \lambda-\gamma, \lambda-2 \gamma]=0$ and we can immediately substitute it in equations (4.4) and (4.5) under the specializations $\lambda_{j}=$ $\lambda-j \gamma$. By doing so we are left with the following relation,

$$
\begin{aligned}
\Lambda(\lambda) \Lambda(\lambda-\gamma) \Lambda(\lambda-2 \gamma)= & \Lambda(\lambda)\left[M_{1}^{(1)}(\lambda-\gamma, \lambda-2 \gamma)+N_{2,1}^{(2)}(\lambda, \lambda-\gamma, \lambda-2 \gamma)\right] \\
& +\Lambda(\lambda-\gamma) M_{2}^{(2)}(\lambda, \lambda-\gamma, \lambda-2 \gamma) \\
& +\Lambda(\lambda-2 \gamma) M_{1}^{(2)}(\lambda, \lambda-\gamma, \lambda-2 \gamma)
\end{aligned}
$$

which simplifies to

$$
\begin{aligned}
\Lambda(\lambda) \Lambda(\lambda-\gamma) \Lambda(\lambda-2 \gamma)= & -\Lambda(\lambda) \prod_{j=1}^{L} \sinh \left(\lambda-\mu_{j}\right) \sinh \left(\lambda-\mu_{j}-2 \gamma\right) \\
& +\Lambda(\lambda-\gamma) 2 \cosh (\gamma) \prod_{j=1}^{L} \sinh \left(\lambda-\mu_{j}\right) \sinh \left(\lambda-\mu_{j}-\gamma\right)
\end{aligned}
$$




$$
-\Lambda(\lambda-2 \gamma) \prod_{j=1}^{L} \sinh \left(\lambda-\mu_{j}+\gamma\right) \sinh \left(\lambda-\mu_{j}-\gamma\right)
$$

upon the use of $(\overline{3.9})$. The representation $(\overline{4.12})$ can now be used in $(4.27)$. In this way we set $\lambda=w_{i}+\gamma$ in $(4.27)$, and for this particular specialization we notice that the term $\left.\Lambda(\lambda-\gamma)\right|_{\lambda=w_{i}+\gamma}$ vanishes. This procedure then yields the following equation determining the set of zeroes $\left\{w_{j}\right\}$,

$$
\prod_{k=1}^{L} \frac{\sinh \left(w_{i}-\mu_{k}+\gamma\right) \sinh \left(w_{i}-\mu_{k}-\gamma\right)}{\sinh \left(w_{i}-\mu_{k}+2 \gamma\right) \sinh \left(w_{i}-\mu_{k}\right)}=-\prod_{j=1}^{L-1} \frac{\sinh \left(w_{j}-w_{i}+\gamma\right)}{\sinh \left(w_{j}-w_{i}-\gamma\right)}
$$

Although the equation (4.28) has been derived using the property $(4.22)$ for the case $l=3$, we notice that $(4.28)$ reduces to $(4.24)$ for values of $\gamma$ belonging to $l=2$. Thus our results so far show that equation $(4.28)$ is valid for for both cases $l=2$ and $l=3$.

$\boldsymbol{l}=4$. This particular root of unity condition also truncates the system of equations (3.8). For $l=4$ we are then left with the relation

$$
\left.\left\{\sum_{m=0}^{2} \sum_{0 \leq i_{1}<\cdots<i_{2 m} \leq 3} V_{i_{2 m}, \ldots, i_{1}}^{(2 m)} \widehat{\prod}_{\lambda}^{i_{1}, \ldots, i_{2 m}}\right\} \Lambda(\lambda)\right|_{\lambda_{j}=\lambda-j \gamma}=0
$$

where the form of the functions $V_{i_{2 m}, \ldots, i_{1}}^{(2 m)}$ are given by $(4.9)$. By making explicit use of $(4.9)$ we find that $(4.29)$ simplifies to

$$
\begin{aligned}
& \Lambda(\lambda) \Lambda(\lambda-\gamma) \Lambda(\lambda-2 \gamma) \Lambda(\lambda-3 \gamma)= \\
& +\Lambda(\lambda-\gamma) \Lambda(\lambda-2 \gamma) \frac{\sinh (3 \gamma)}{\sinh (\gamma)} \prod_{k=1}^{L} \sinh \left(\lambda-\mu_{k}\right) \sinh \left(\lambda-\mu_{k}-2 \gamma\right) \\
& -\Lambda(\lambda-2 \gamma) \Lambda(\lambda-3 \gamma) \prod_{k=1}^{L} \sinh \left(\lambda-\mu_{k}+\gamma\right) \sinh \left(\lambda-\mu_{k}-\gamma\right) \\
& -\Lambda(\lambda) \Lambda(\lambda-\gamma) \prod_{k=1}^{L} \sinh \left(\lambda-\mu_{k}-\gamma\right) \sinh \left(\lambda-\mu_{k}-3 \gamma\right) \\
& -\Lambda(\lambda) \Lambda(\lambda-3 \gamma) \prod_{k=1}^{L} \sinh \left(\lambda-\mu_{k}\right) \sinh \left(\lambda-\mu_{k}-2 \gamma\right)+\mathcal{Q}(\lambda),
\end{aligned}
$$


where the function $\mathcal{Q}(\lambda)$ is given by

$$
\begin{aligned}
\mathcal{Q}(\lambda)= & \frac{\sinh (3 \gamma)}{\sinh (\gamma)} \prod_{k=1}^{L} \sinh \left(\lambda-\mu_{k}\right)^{2} \sinh \left(\lambda-\mu_{k}-2 \gamma\right)^{2} \\
& -\prod_{k=1}^{L} \sinh \left(\lambda-\mu_{k}+\gamma\right) \sinh \left(\lambda-\mu_{k}-3 \gamma\right) \sinh \left(\lambda-\mu_{k}-\gamma\right)^{2} \\
& -2 \cosh (2 \gamma) \prod_{k=1}^{L} \sinh \left(\lambda-\mu_{k}\right) \sinh \left(\lambda-\mu_{k}-2 \gamma\right) \sinh \left(\lambda-\mu_{k}-\gamma\right)^{2}
\end{aligned}
$$

In what follows we shall describe how one can extract a set of equations determining the set of zeroes $\left\{w_{j}\right\}$ from the functional relation $(4.30)$. For that we assume the representation (4.12) and set $\lambda=w_{i}+\gamma$ in (4.30). Under this specialization the term $\left.\Lambda(\lambda-\gamma)\right|_{\lambda=w_{i}+\gamma}$ vanishes and we are left with a relation depending on the function $\mathcal{Q}\left(w_{i}+\gamma\right)$. Next we consider the specialization $\lambda=w_{i}+2 \gamma$ such that $\left.\Lambda(\lambda-2 \gamma)\right|_{\lambda=w_{i}+2 \gamma}=$ 0 . This procedure yields two equations: one involving the function $\mathcal{Q}\left(w_{i}+\gamma\right)$ and another depending on $\mathcal{Q}\left(w_{i}+2 \gamma\right)$. However, we can readily verify that $\mathcal{Q}(\lambda)=\mathcal{Q}(\lambda+\gamma)$ under the root of unity condition $l=4$. This property allows us to eliminate the functions $\mathcal{Q}$ from our equations leaving us with the following relation,

$$
\prod_{k=1}^{L} \frac{\sinh \left(w_{i}-\mu_{k}+\gamma\right) \sinh \left(w_{i}-\mu_{k}-\gamma\right)}{\sinh \left(w_{i}-\mu_{k}+2 \gamma\right) \sinh \left(w_{i}-\mu_{k}\right)}=-\frac{\Lambda\left(w_{i}-\gamma\right)}{\Lambda\left(w_{i}+\gamma\right)}
$$

By substituting the representation $(4.12)$ into $(4.32)$ we immediately recognize equation (4.28). Thus our analysis so far shows that the set of equations (4.28) is valid for $l=2,3,4$. In fact, Eq. (4.28) seems to be valid for arbitrary roots of unity as we shall discuss.

General case. For arbitrary values of $l$ the property (4.22) truncates the system of functional relations (3.8) and we only need to consider the following equation,

$$
\left.\left\{\sum_{m=0}^{[l] / 2} \sum_{0 \leq i_{1}<\cdots<i_{2 m} \leq l-1} V_{i_{2 m}, \ldots, i_{1}}^{(2 m)} \widehat{\prod}_{\lambda}^{i_{1}, \ldots, i_{2 m}}\right\} \Lambda(\lambda)\right|_{\lambda_{j}=\lambda-j \gamma}=0 .
$$

Then we assume the representation (4.12) and consider the sequence of specializations $\lambda=w_{i}+p \gamma$ for $1 \leq p \leq p-2$. This procedure yields one equation at each level of specialization. Similarly to the case $l=4$, the resulting system of equations can be manipulated in order to find compact equations determining the roots $w_{j}$. Although this last step involves the use of non-trivial properties satisfied by the functions $V_{i_{2 m}, \ldots, i_{1}}^{(2 m)}$, the procedure above described holds in general and its implementation for particular values of $l$ leads to the very same equation (4.28). A rigorous proof of $(4.28)$ for arbitrary values of $l$ is still missing but our analysis so far leads us to conjecture that (4.28) is valid for general roots of unity. 


\section{Concluding remarks}

The main result of this work is the formula $(4.8)$ which states a relation between the six-vertex model with DWBC and the anti-periodic Heisenberg chain. This relation is a direct consequence of the algebraic-functional approach introduced in $[13$ and refined in the series of works $[16-18]$. The Yang-Baxter algebra is the main ingredient for the derivation of (4.8) which allows us to establish a very non-trivial relation between the zeroes of certain quantities related to six-vertex models with different boundary conditions. In one hand we have the zeroes of the partition function of the six-vertex model with DWBC. On the other hand, we have the zeroes of the transfer matrix eigenvalues associated with the six-vertex model with a non-diagonal boundary twist. The relation between those zeroes is then precised in (4.15) and (4.17).

In this work we have also analyzed the cases where the six-vertex model anisotropy parameter satisfies a root of unity condition. In that case we have found a compact set of equations, namely (4.28), characterizing the zeroes of the eigenvalues $\Lambda$.

Boundary conditions of domain wall type can be formulated for a variety of lattice integrable systems. In particular, the so called $8 \mathrm{~V}$-SOS model also admits domain wall boundary conditions and it has been studied through this algebraic-functional approach in $[17,18]$. The latter consists of an elliptic integrable system and one might wonder if there exists a twisted transfer matrix such that a relation analogous to (4.8) holds. This problem has eluded us so far and its investigation would probably bring further insights into the structure of integrable solid-on-solid models.

\section{Acknowledgements}

The author thanks G. Arutyunov for very useful discussions. This work is supported by the Netherlands Organization for Scientific Research (NWO) under the VICI grant 680-47-602 and by the ERC Advanced grant research programme No. 246974, "Supersymmetry: a window to non-perturbative physics". The author also thanks the D-ITP consortium, a program of the Netherlands Organization for Scientific Research (NWO) funded by the Dutch Ministry of Education, Culture and Science (OCW).

\section{A The function $V_{i_{2 m}, \ldots, i_{1}}^{(2 m)}$}

In this appendix we aim to discuss the derivation of formulae $(4.8)$ and $(4.9)$. The function $V_{i_{2 m}, \ldots, i_{1}}^{(2 m)}$ given by (4.9) follows straightforwardly from the functions $M_{i}^{(n)}$ and $N_{j, i}^{(n)}$ defined in (3.9). We shall start by reviewing the cases $L=2$ and $L=3$ already discussed in Section 4 .

$\boldsymbol{L}=\mathbf{2}$. The first equation of $(4.3)$ can be rewritten as

$$
Z\left(X^{0,1}\right) k_{0}=\Lambda\left(\lambda_{0}\right) \Lambda\left(\lambda_{1}\right)-M_{1}^{(1)}\left(\vec{X}^{0,1}\right)
$$


and we can compare its RHS with (4.10). In this way we require that $V_{1,0}^{(2)}=-M_{1}^{(1)}\left(\vec{X}^{0,1}\right)$. The explicit evaluation of (4.9) then yields the following expression

$$
V_{1,0}^{(2)}=\frac{c\left(\lambda_{0}-\lambda_{1}\right)}{b\left(\lambda_{0}-\lambda_{1}\right)} \prod_{k=1}^{2} a\left(\lambda_{0}-\mu_{k}\right) b\left(\lambda_{1}-\mu_{k}\right)+\frac{c\left(\lambda_{1}-\lambda_{0}\right)}{b\left(\lambda_{1}-\lambda_{0}\right)} \prod_{k=1}^{2} a\left(\lambda_{1}-\mu_{k}\right) b\left(\lambda_{0}-\mu_{k}\right),
$$

which corresponds to $-M_{1}^{(1)}\left(\vec{X}^{0,1}\right)$ according to $(3.9)$.

$\boldsymbol{L}=3$. Similarly to the previous case, we firstly rewrite the first equation of $(4.5)$ as

$$
\begin{aligned}
Z\left(X^{0,2}\right) k_{0}= & \Lambda\left(\lambda_{0}\right) \Lambda\left(\lambda_{1}\right) \Lambda\left(\lambda_{2}\right)-\Lambda\left(\lambda_{0}\right)\left[M_{1}^{(1)}\left(\vec{X}^{1,2}\right)+N_{2,1}^{(2)}\left(\vec{X}^{0,2}\right)\right] \\
& -\Lambda\left(\lambda_{1}\right) M_{2}^{(2)}\left(\vec{X}^{0,2}\right)-\Lambda\left(\lambda_{2}\right) M_{1}^{(2)}\left(\vec{X}^{0,2}\right) .
\end{aligned}
$$

We can now compare the RHS of $(\mathrm{A} .2)$ with $(4.11)$. By doing so we find the following conditions:

$$
\begin{aligned}
& V_{1,0}^{(2)}=-M_{1}^{(2)}\left(\vec{X}^{0,2}\right) \\
& V_{2,0}^{(2)}=-M_{2}^{(2)}\left(\vec{X}^{0,2}\right) \\
& V_{2,1}^{(2)}=-M_{1}^{(1)}\left(\vec{X}^{1,2}\right)-N_{2,1}^{(2)}\left(\vec{X}^{0,2}\right) .
\end{aligned}
$$

It is now a straightforward computation to verify that the functions $V_{1,0}^{(2)}, V_{2,0}^{(2)}$ and $V_{2,1}^{(2)}$ obtained from (4.9) satisfy the conditions (A.3) with functions $M_{i}^{(n)}$ and $N_{j, i}^{(n)}$ given by (3.9). It is also worth remarking that the verification of the third condition of (A.3) involves the simplification of functions as it typically occurs in algebraic Bethe ansatz framework.

$\boldsymbol{L}=4$. We start from $(3.8)$ and for the case $L=4$ we set $n=0,1,2,3,4$. In this way we are left with a total of 5 equations for the functions $\mathcal{F}_{n}$ which can be solved in favor of the eigenvalue $\Lambda$. The resulting equation will then depend on the functions $M_{i}^{(n)}$ and $N_{j, i}^{(n)}$ defined in (3.9), and it can be directly compared with (4.8). By doing so we find the following conditions,

$$
\begin{array}{ll}
V_{3,0}^{(2)}=-M_{3}^{(3)}\left(\vec{X}^{0,3}\right) & V_{3,2}^{(2)}=-M_{1}^{(1)}\left(\vec{X}^{2,3}\right)-N_{2,1}^{(2)}\left(\vec{X}^{1,3}\right)-N_{3,2}^{(3)}\left(\vec{X}^{0,3}\right) \\
V_{2,0}^{(2)}=-M_{2}^{(3)}\left(\vec{X}^{0,3}\right) & V_{3,1}^{(2)}=-M_{2}^{(2)}\left(\vec{X}^{1,3}\right)-N_{3,1}^{(3)}\left(\vec{X}^{0,3}\right) \\
V_{1,0}^{(2)}=-M_{1}^{(3)}\left(\vec{X}^{0,3}\right) & V_{2,1}^{(2)}=-M_{1}^{(2)}\left(\vec{X}^{1,3}\right)-N_{2,1}^{(3)}\left(\vec{X}^{0,3}\right)
\end{array}
$$

in addition to

$$
\begin{aligned}
V_{3,2,1,0}^{(4)}= & M_{1}^{(3)}\left(\vec{X}^{0,3}\right) M_{1}^{(1)}\left(\vec{X}_{1}^{1,3}\right)+M_{2}^{(3)}\left(\vec{X}^{0,3}\right) M_{1}^{(1)}\left(\vec{X}_{2}^{1,3}\right)+M_{3}^{(3)}\left(\vec{X}^{0,3}\right) M_{1}^{(1)}\left(\vec{X}_{3}^{1,3}\right) \\
& +N_{2,1}^{(3)}\left(\vec{X}^{0,3}\right) M_{1}^{(1)}\left(\vec{X}_{1,2}^{0,3}\right)+N_{3,1}^{(3)}\left(\vec{X}^{0,3}\right) M_{1}^{(1)}\left(\vec{X}_{0,3}^{0,3}\right)+N_{3,2}^{(3)}\left(\vec{X}^{0,3}\right) M_{1}^{(1)}\left(\vec{X}_{2,3}^{0,3}\right)
\end{aligned}
$$

Both relations $(\overline{\mathrm{A} .4})$ and $(\overline{\mathrm{A} .5})$ can be readily verified with the help of $(\overline{4.9})$ and $(\overline{3.9})$. 
General $\boldsymbol{L}$. The structure of the function $V_{i_{2 m}, \ldots, i_{1}}^{(2 m)}$ for arbitrary values of $L$ is obtained from particular combinations of the functions $M_{i}^{(n)}$ and $N_{j, i}^{(n)}$. These combinations are built by eliminating the functions $\mathcal{F}_{n}$ from the system of equations (3.8) in favor of the eigenvalue $\Lambda$. By carrying out this procedure we find the relation (4.8). The function $V_{i_{2 m}, \ldots, i_{1}}^{(2 m)}$, as defined in $(4.9)$, captures the aforementioned combinations of $M_{i}^{(n)}$ and $N_{j, i}^{(n)}$ which are explicitly given by (3.9). Although it is a cumbersome computation, the simplifications required to arrive at formula (4.9) are performed in much the same spirit of the algebraic Bethe ansatz.

\section{References}

[1] E. H. Lieb. Residual entropy of square lattice. Phys. Rev., 162(1):162, 1967.

[2] R. J. Baxter. Eight vertex model in lattice statistics. Phys. Rev. Lett., 26:832, 1971.

[3] V. G. Knizhnik and A. B. Zamolodchikov. Current algebra and Wess-Zumino model in two dimensions. Nucl. Phys. B, 247(1):83-103, 1984.

[4] V. E. Korepin. Calculation of norms of Bethe wave functions. Commun. Math. Phys., 86:391-418, 1982.

[5] R. J. Baxter. Three-colorings of the square lattice: A hard squares model. J. Math. Phys., 11(10):3116, 1970.

[6] O. Foda, M. Wheeler, and M. Zuparic. Domain wall partition functions and KP. J. Stat. Mech., 2009.

[7] A. Lascoux. The 6 Vertex Model and Schubert Polynomials. SIGMA, 3:029, 2007.

[8] H. Rosengren. The three-colour model with domain wall boundary conditions. Adv. Appl. Math., 46:481, 2011.

[9] G. Kuperberg. Another proof of the alternating sign matrix conjecture. Inter. Math. Res. Notes, (3):139-150, 1996.

[10] W. Galleas. Functional relations and the Yang-Baxter algebra. Journal of Physics: Conference Series, 474:012020, 2013.

[11] H. J. de Vega. Families of commuting transfer matrices and integrable models with disorder. Nucl. Phys. B, 240(4):495-513, 1984.

[12] M. T. Batchelor, R. J. Baxter, M. J. Orourke, and C. M. Yung. Exact solution and interfacial tension of the 6-vertex model with antiperiodic boundary conditions. $J$. Phys. A - Math. and Gen., 28(10):2759-2770, 1995.

[13] W. Galleas. Functional relations from the Yang-Baxter algebra: Eigenvalues of the $X X Z$ model with non-diagonal twisted and open boundary conditions. Nucl. Phys. $B, 790(3): 524-542,2008$. 
[14] G. Niccoli. Antiperiodic spin- $1 / 2 X X Z$ quantum chains by separation of variables: Complete spectrum and form factors. Nucl. Phys. B, 870:397-420, 2013.

[15] J. Cao, W. L. Yang, K. Shi, and Y. Wang. Off-diagonal Bethe ansatz and exact solution of a topological spin ring. Phys. Rev. Lett., 111:137201, 2013.

[16] W. Galleas. Functional relations for the six-vertex model with domain wall boundary conditions. J. Stat. Mech., (06):P06008, 2010.

[17] W. Galleas. Multiple integral representation for the trigonometric SOS model with domain wall boundaries. Nucl. Phys. B, 858(1):117-141, 2012, math-ph/1111.6683.

[18] W. Galleas. Refined functional relations for the elliptic SOS model. Nucl. Phys. B, $867: 855-871,2013$.

[19] R. J. Baxter. Exactly Solved Models in Statistical Mechanics. Dover Publications, Inc., Mineola, New York, 2007.

[20] A. G. Izergin. Statistical sum of the six-vertex model in a finite lattice. Sov. Phys. Dokl., 32:878, 1987.

[21] W. Galleas. Scalar product of Bethe vectors from functional equations. Comm. Math. Phys., 329(1):141-167, 2014.

[22] J. de Gier, W. Galleas, and M. Sorrell. Multiple integral formula for the off-shell six vertex scalar product. 2011, hep-th/1111.3712.

[23] V. O. Tarasov. On Bethe vectors for the $X X Z$ model at roots of unity. Journal of Mathematical Sciences, 125(2):242-248, 2005.

[24] Y. G. Stroganov. A new calculation method for partition functions in some lattice models. Phys. Lett. A, 74:116, 1979. 